\title{
Error estimation for boundary element method
}

\author{
M.T. Liang*, J.T. Chen, S.S. Yang \\ Department of Harbor and River Engineering, Taiwan Ocean University, Keelung, Taiwan
}

Received 21 January 1998; accepted 7 August 1998

\begin{abstract}
In this paper, six error indicators obtained from dual boundary integral equations are used for local estimation, which is an essential ingredient for all adaptive mesh schemes in BEM. Computational experiments are carried out for the two-dimensional Laplace equation. The curves of all these six error estimators are in good agreement with the shape of the error curve. The results show that the adaptive mesh based on any one of these six error indicators converges faster than does equal mesh discretization. (C) 1999 Elsevier Science Ltd. All rights reserved.
\end{abstract}

Keywords: Dual boundary integral; Error estimator; Adaptive technique

\section{Introduction}

The numerical method can be utilized to solve the governing equation of a problem especially when the exact solution is not easy to obtain. However, the discretization process, which transforms a continuous system into a discrete system with finite number of degrees of freedom, results in errors. The discretization error is defined as the difference between the exact solution and the numerical approximation of the governing equation. Obtaining a reliable error estimation [1-12] is very important in order to guarantee a certain level of accuracy of the numerical result, and is a key factor of the adaptive mesh procedure [3-6,1317]. Thus, estimation of the discretization error in the Boundary Element Method (BEM) is worthy of study.

The h-refinement $[5,6]$, p-refinement $[5,18]$ and r-refinement schemes [5] have been recently used to improve numerical accuracy. In the h-refinement scheme, the total number of elements increase, but the order of the interpolation function remains unchanged. As the global matrix must be reformulated after mesh refinement, the computational cost becomes very high. In this way, the efficient remesh tactics are required when h-refinement scheme is adopted. The adaptive tactics for h-refinement are generally referred to as the reference value method [5], in which the element mesh is refined where the error is larger than the prescribed reference value. This method provides a facile error criterion to determine which elements should be divided into

\footnotetext{
* Corresponding author. Fax: + 886-2-2462-2192-6102.
}

more partitions by considering the integral equation at the sampling point.

A large number of studies on adaptive BEM have been done by Kamiya et al. [4] using sample point error estimation. However, the error stems not only from the discretization procedure, but also from the mismatch of the collocation points on the boundary. Zarikian et al. [7] and Paulino et al. [8,9] used pointwise error estimation to study the convergency of the interior problem by using the dual BEM. Both the first (singular integral equation) and second (hypersingular integral equation) kind of formulation of BEM can independently determine the unknown boundary data for the problem without degenerate boundary [19]. The difference in the solutions obtained from these two methods can be used as an index of error estimation. This provides a new guide for remeshing without the mismatch of the collocation points on the boundary in the sample point error method. By creating more divisions in the boundary mesh where the estimated error is large, the exact error will be reduced more efficiently.

In this paper, dual boundary integral equations are utilized to find a reliable error estimator. The estimated error is defined as the residue [10]. The residue is based on the imbalance of the energy, which is calculated by substituting the solution of the unknown boundary data obtained from the first kind of direct BEM previously and known boundary data into the hypersingular integral representation. Two numerical examples are performed for twodimensional problems of the Laplace equation with the Dirichlet and mixed boundary conditions using the BEPO2D program [19]. 


\section{Review of a posteriori pointwise error estimator for the boundary element method}

Considering a two-dimensional Laplace equation, as the one below,

$\nabla^{2} u(x)=0, x \in D$

where $D$ is the domain, and $u(x)$ is the potential function, the first kind of BEM (direct BEM) for this problem can be written as

$\pi u(x)=C . P . V . \int_{B} T(s, x) u(s) d B(s)-\int_{B} U(s, x) t(s) d B(s)$,

$x \in B$,

where $B$ is the boundary and $U(s, x)$ is a fundamental solution and can be expressed as

$U(s, x)=\ln r$,

in which $r$ is the distance between $x$ and $s, T(s, x)$ is the directional derivative in the $n_{\mathrm{s}}$ direction, where $n_{\mathrm{s}}$ is the outnormal vector at $s$ i.e. $T(s, x)=\frac{\partial U(s, x)}{\partial n_{s}} ; t(x)$ is the flux i.e. $t(x)=\frac{\partial u(x)}{\partial n_{x}}$ where $n_{x}$ is the outnormal vector at $x$ and C.P.V. means the Cauchy Principal Value. Taking the normal derivative of Eq. (2) with respect to the $n_{x}$ direction results in the second kind representation of BEM (hypersingular integral equation) as follows:

$$
\begin{aligned}
\pi t(x)= & \text { H.P.V. } \int_{B} M(s, x) u(s) d B(s) \\
& - \text { C.P.V. } \int_{B} L(s, x) t(s) d B(s), x \in B,
\end{aligned}
$$

where $L(s, x)$ is the directional derivative in the $n_{\mathrm{x}}$ direction of $U(s, x)$; (i.e. $\left.L(s, x)=\partial U(s, x) / \partial n_{x}\right) M(s, x)$ is the directional derivative in the $n_{\mathrm{x}}$ direction of $T(s, x)$; i.e. $\left.M(s, x)=\partial^{2} U(s, x) / \partial n_{x} \partial n_{s}\right)$ and H.P.V. denotes the Hadamard Principal Value.

For an interior problem, Paulino et al. [8,9] employed the direct BEM to solve the unknown boundary data, $u_{\mathrm{UT}}$, in the Neumann problem and $t_{\mathrm{UT}}$ in the Dirichlet problem, and then substituted $u_{\mathrm{UT}}$ or $t_{\mathrm{UT}}$ into the hypersingular integral equation. Inequality occurs in Eq. (4) as follows:

$$
\begin{aligned}
\pi t_{U T}(x) \neq & \text { H.P.V. } \int_{\tilde{B}} M(s, x) u_{U T}(s) d B(s) \\
& - \text { C.P.V. } \int_{\tilde{B}} L(s, x) t_{U T}(s) d B(s), x \in \tilde{B},
\end{aligned}
$$

where $\tilde{B}$ is the approximate boundary after discretization of $B$. One can define the residue $\epsilon(x)$ as the unbalance energy term,

$$
\begin{aligned}
\varepsilon(x)= & -\pi t_{U T}(x)+H . P . V . \int_{\tilde{B}} M(s, x) u_{U T}(s) d B(s) \\
& - \text { C.P.V. } \int_{\tilde{B}} L(s, x) t_{U T}(s) d B(s), x \in \tilde{B} .
\end{aligned}
$$

The unequal relationship in Eq. (5) indicates that the exact solution has not been obtained. If $u_{\mathrm{e}}(s)$ and $\mathrm{t}_{\mathrm{e}}(s)$ represent the exact solution, which is assumed to also be valid everywhere on the approximate boundary $\tilde{B}$ i.e., the discretization error is neglected, then

$$
\begin{aligned}
0= & -\pi t_{e}(x)+H . P . V . \int_{\tilde{B}} M(s, x) u_{e}(s) d B(s) \\
& - \text { C.P.V. } \int_{\tilde{B}} L(s, x) t_{e}(s) d B(s), x \in \tilde{B} .
\end{aligned}
$$

Substracting Eq. (7) from Eq. (6), we obtain

$$
\begin{aligned}
\varepsilon(x)= & -\pi\left\{t_{U T}(x)-t_{e}(x)\right\}+H . P . V . \int_{\tilde{B}} M(s, x)\left\{u_{U T}(s)\right. \\
& \left.-u_{e}(s)\right\} d B(s) \\
& - \text { C.P.V. } \int_{\tilde{B}} L(s, x)\left\{t_{U T}(s)-t_{e}(s)\right\} d B(s), x \in \tilde{B} .
\end{aligned}
$$

Define the notations

$$
\begin{aligned}
& E_{u}(s)=u_{U T}(s)-u_{e}(s) ; E_{t}(s)=t_{U T}(s)-t_{e}(s) \\
& E_{t}(x)=t_{U T}(x)-t_{e}(x)
\end{aligned}
$$

For a Dirichlet problem, $u_{\mathrm{e}}$ is equal to $u_{\mathrm{UT}}$ on the specified boundary; then, Eq. (8) can be reduced to

$$
\begin{aligned}
\varepsilon(x)= & -\pi\left\{t_{U T}(x)-t_{e}(x)\right\} \\
& - \text { C.P.V. } \int_{\tilde{B}} L(s, x)\left\{t_{U T}(s)-t_{e}(s)\right\} d B(s), x \in \tilde{B} .
\end{aligned}
$$

In contrast, for a Neumann problem, $t_{\mathrm{e}}$ is equal to $t_{\mathrm{UT}}$ on the specified boundary, and Eq. (8) can be expressed as

$$
\begin{aligned}
\varepsilon(x)= & -\pi\left\{t_{U T}(x)-t_{e}(x)\right\}+H . P . V . \int_{\tilde{B}} M(s, x)\left\{u_{U T}(s)\right. \\
& \left.-u_{e}(s)\right\} d B(s), x \in \tilde{B} .
\end{aligned}
$$

\section{New version of a posteriori pointwise error estimator for the boundary element method}

The first and second kind of the direct BEM formulation provide a good basis for evaluating the discretization error on the boundary since both equations can yield an approximate solution. Dual boundary integral equations provide us 


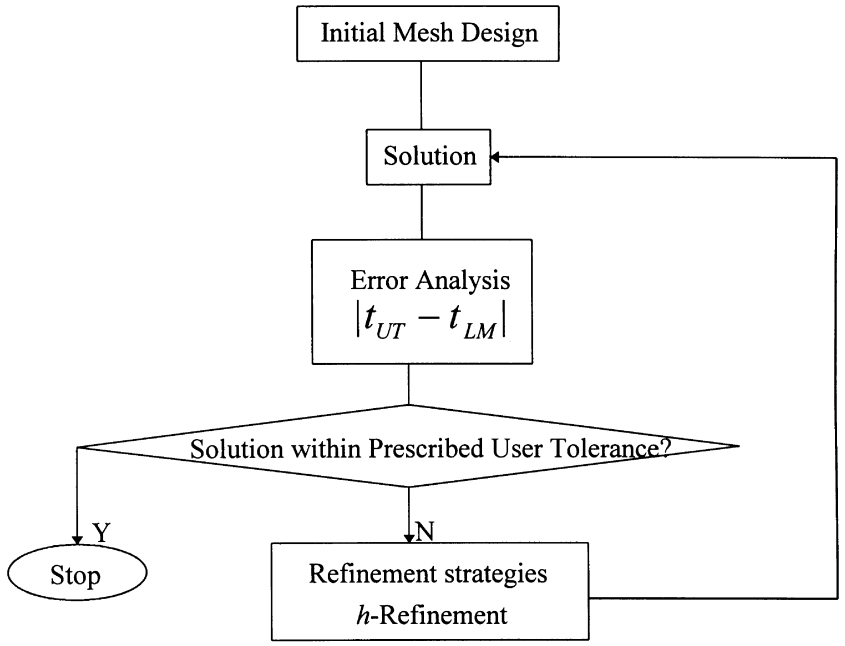

Fig. 1. Flowchart of self adaptive mesh refinement.

with two constraints for all the boundary data [20]; the first and second kind of BEM formulations are shown as follows:

$$
\begin{aligned}
\pi u_{U T}(x)= & \text { C.P.V. } \int_{\tilde{B}} T(s, x) u_{U T}(s) d B(s) \\
& -\int_{\tilde{B}} U(s, x) t_{U T}(s) d B(s), x \in \tilde{B} \\
\pi t_{L M}(x)= & \text { H.P.V. } \int_{\tilde{B}} M(s, x) u_{L M}(s) d B(s) \\
& - \text { C.P.V. } \int_{\tilde{B}} L(s, x) t_{L M}(s) d B(s), x \in \tilde{B} .
\end{aligned}
$$

Define $\left|u_{U T}(x)-u_{L M}(x)\right|$ and $\left|t_{U T}(x)-t_{L M}(x)\right|$ as a pair of new error estimators. We investigate this new error estimator $\left|u_{U T}(x)-u_{L M}(x)\right|$ or $\left|t_{U T}(x)-t_{L M}(x)\right|$ for comparison with both the error estimator $\epsilon(x)$ and exact error $E(\mathrm{x})$ defined by Paulino et al. [8,9].

According to the definition, $\epsilon(x)$ in [8] is

$$
\begin{aligned}
\varepsilon(x)= & -\pi t_{U T}(x)+H . P . V . \int_{\tilde{B}} M(s, x) u_{U T}(s) d B(s) \\
& - \text { C.P.V. } \int_{\tilde{B}} L(s, x) t_{U T}(s) d B(s), x \in \tilde{B} .
\end{aligned}
$$

For a Dirichlet problem, Eq. (14) can be rewritten as

$$
\begin{aligned}
\varepsilon(x)= & -\pi t_{U T}(x)+H . P . V . \int_{\tilde{B}} M(s, x) u_{U T}(s) d B(s) \\
& - \text { C.P.V. } \int_{\tilde{B}} L(s, x) t_{U T}(s) d B(s) \\
& + \text { C.P.V. } \int_{\tilde{B}} L(s, x) t_{L M}(s) d B(s) \\
& - \text { C.P.V. } \int_{\tilde{B}} L(s, x) t_{L M}(s) d B(s), x \in \tilde{B}
\end{aligned}
$$

after an addition and subtraction technique. Using Eq. (13), Eq. (15) becomes

$$
\begin{aligned}
\varepsilon(x)= & -\pi\left\{t_{U T}(x)-t_{L M}(x)\right\} \\
& - \text { C.P.V. } \int_{\tilde{B}} L(s, x)\left\{t_{U T}(s)-t_{L M}(s)\right\} d B(s), x \in \tilde{B} .
\end{aligned}
$$

For a Neumann problem, Eq. (14) can be similarly rewritten as

$$
\begin{aligned}
\varepsilon(x)= & -\pi t_{U T}(x)+H . P . V . \int_{\tilde{B}} M(s, x) u_{U T}(s) d B(s) \\
& - \text { C.P.V. } \int_{\tilde{B}} L(s, x) t_{U T}(s) d B(s) \\
& + \text { H.P.V. } \int_{\tilde{B}} M(s, x) u_{L M}(s) d B(s) \\
& - \text { H.P.V. } \int_{\tilde{B}} M(s, x) u_{L M}(s) d B(s), x \in \tilde{B} .
\end{aligned}
$$

Using Eq. (13), Eq. (17) yields

$$
\begin{aligned}
\varepsilon(x)= & -\pi\left\{t_{U T}(x)-t_{L M}(x)\right\} \\
& + \text { H.P.V. } \int_{\tilde{B}} M(s, x)\left\{u_{U T}(s)-u_{L M}(s)\right\} d B(s), x \in \tilde{B} .
\end{aligned}
$$

Comparing both Eq. (10) with Eq. (16) and Eq. (11) with Eq. (18), it can be found that $E_{\mathrm{t}}(x)$ and $\mathrm{E}_{\mathrm{u}}(x)$ as defined by Paulino et al. [8,9] are, respectively, $\left\{t_{U T}(x)-t_{L M}(x)\right\}$ and $\left\{u_{U T}(x)-u_{L M}(x)\right\}$, if the discretization procedure is employed. 


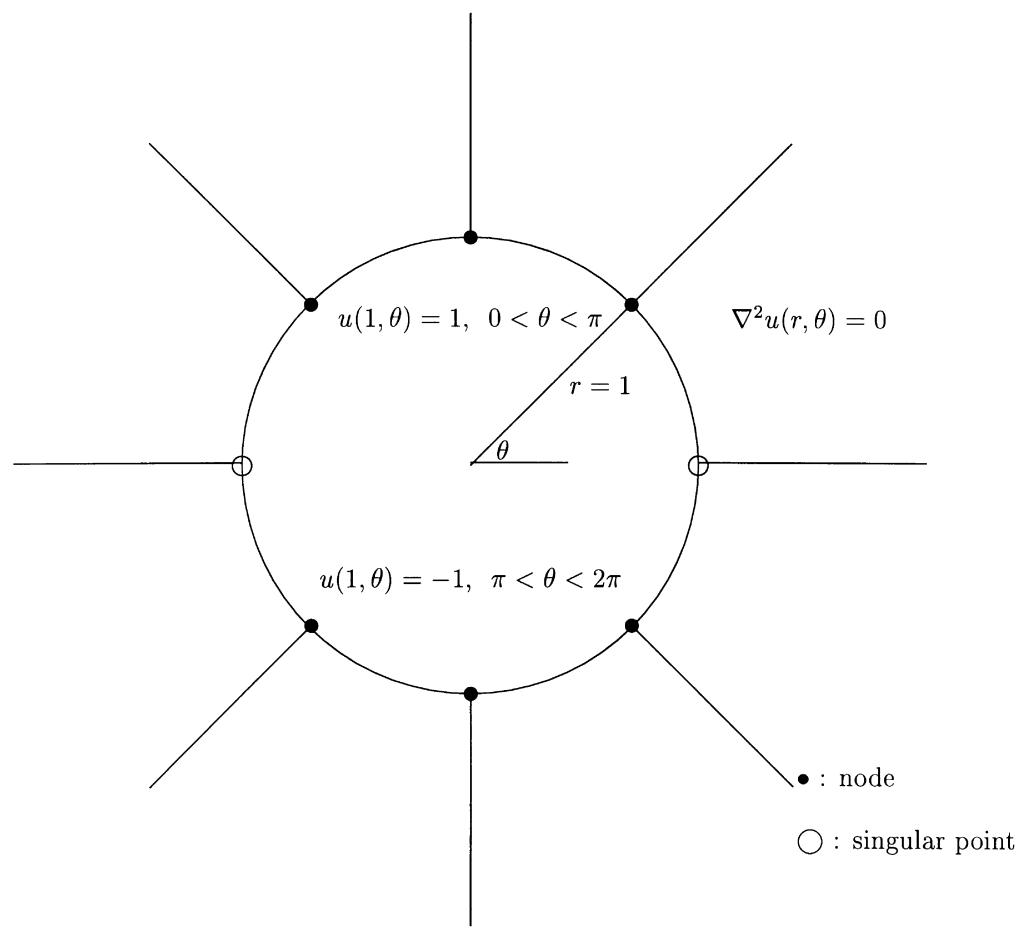

Fig. 2. The initial element mesh for the exterior problem.

\section{Six types of error estimators}

In this section, we shall summarize six kinds of error estimators using the dual boundary integral formulation.

I. The proposed error estimate, $\epsilon(x)$, is defined as the error which arises when the approximate solution obtained from the $U T$ method is substituted into Eq. (4), as shown below:

$$
\begin{aligned}
\varepsilon(x)= & -\pi t_{U T}(x)+H . P . V . \int_{\tilde{B}} M(s, x) u_{U T}(s) d B(s) \\
& - \text { C.P.V. } \int_{\tilde{B}} L(s, x) t_{U T}(s) d B(s), x \in \tilde{B} .
\end{aligned}
$$

II. The exact error, $E_{\mathrm{u}}(x)$ or $\mathrm{E}_{\mathrm{t}}(x)$, defined by Paulino et al. $[8,9]$, which has been verified to be $\left|u_{U T}(x)-u_{L M}(x)\right|$ or $\left|t_{U T}(x)-t_{L M}(x)\right|$ is obtained by Eq. (8):

$$
\begin{aligned}
\varepsilon(x)= & -\pi E_{t}(x)+H . P . V . \int_{\tilde{B}} M(s, x) E_{u}(s) d B(s) \\
& - \text { C.P.V. } \int_{\tilde{B}} L(s, x) E_{t}(s) d B(s), x \in \tilde{B} .
\end{aligned}
$$

As $\epsilon(x)$ is specified by Eq. (19), $E_{\mathrm{u}}(x)$ and $\mathrm{E}_{\mathrm{t}}(x)$ can be computed.

III. By using $\left\{t_{U T}(x)-t_{L M}(x)\right\}$ instead of $\epsilon(x)$ in Eq. (20), the modified exact error, $E_{u}^{M}$, in the Neumann problem and $E_{t}^{M}$ in the Dirichlet problem can be obtained by the following expression:

$$
\begin{aligned}
& \left\{t_{U T}(x)-t_{L M}(x)\right\} \\
& =-\pi E_{t}^{M}(x)+H . P . V . \int_{\tilde{B}} M(s, x) E_{u}^{M}(s) d B(s) \\
& \quad-\text { C.P.V. } \int_{\tilde{B}} L(s, x) E_{t}^{M}(s) d B(s), x \in \tilde{B}
\end{aligned}
$$

since $\left\{t_{U T}(x)-t_{L M}(x)\right\}$ can be specified by the $U T$ method and the $L M$ method.

It is difficult to derive a mathematical relationship between the exact and estimated error, but it is easy to calculate the error between the exact solution and the solution determined by the dual boundary integral formulation if the problem has a closed-form solution.

IV. If the exact solution for the problem is available, a comparison can be made between the exact and estimated error. The pointwise error estimator is defined as $\mid t_{U T}(x)-$ $t_{e}(x) \mid$ for the Dirichlet problem and $\left|u_{U T}(x)-u_{e}(x)\right|$ for the Neumann problem, respectively.

V. $E_{U T}^{A}$ represents the error which replaces the exact solution in the $U T$ method, as follows:

$$
\begin{aligned}
E_{U T}^{A}(x)= & -\pi\left\{t_{U T}(x)-t_{e}(x)\right\}+H . P . V . \int_{B} M(s, x)\left\{u_{U T}(s)\right. \\
& \left.-u_{e}(s)\right\} d B(s) \\
& - \text { C.P.V. } \int_{B} L(s, x)\left\{t_{U T}(s)-t_{e}(s)\right\} d B(s), x \in \tilde{B} .
\end{aligned}
$$




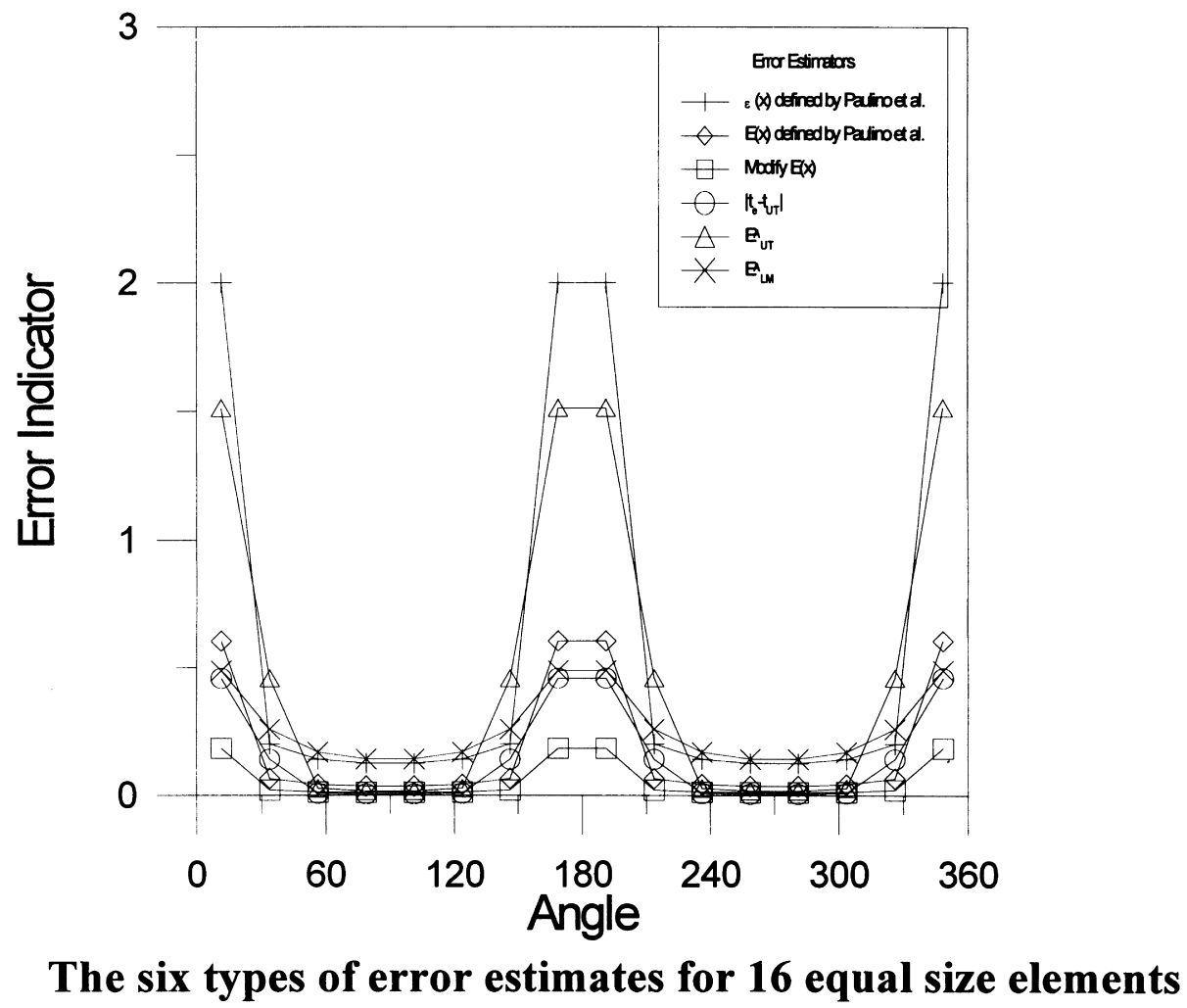

Fig. 3. The six types of error estimators.

VI. $E_{L M}^{A}$ denotes the error which replaces the exact solution in the $L M$ method, as follows:

$$
\begin{aligned}
E_{L M}^{A}(x)= & -\pi\left\{t_{L M}(x)-t_{e}(x)\right\}+H . P . V . \int_{B} M(s, x)\left\{u_{L M}(s)\right. \\
& \left.-u_{e}(s)\right\} d B(s)-\text { C.P.V. } \int_{B} L(s, x)\left\{t_{L M}(s)\right. \\
& \left.-t_{e}(s)\right\} d B(s), x \in \tilde{B} .
\end{aligned}
$$

All of the above six error estimators will be examined to deal with the error curve.

\section{Adaptive tactics and measure of errors}

The role of the adaptive tactical procedure is to determine the region where the elements should be refined. This algorithm is very strongly dependent on error estimation and the mesh refinement scheme. The reference value, error convergency and equilibrium criterion methods [5] have been popular adaptive tactics for the h-refinement scheme [36]. In the case of the reference value method, elements are refined when their errors are larger than the prescribed reference value. Denoting the error at the element $i$ by $\lambda_{\mathrm{i}}$, the reference value $\bar{\lambda}$ may be defined as

$\bar{\lambda}=$ Average of $\lambda_{i}$ or

$\bar{\lambda}=\eta \times \max \left(\lambda_{i}\right)(0<\eta<1)$.

In this paper, the former value in Eq. (24) is chosen because in the latter value in Eq. (25), it is difficult to specify the value of $\eta$ adequately.

As the error estimator and adaptive tactics can be obtained, the self adaptive mesh refinement process can be implemented. The flowchart of the self adaptive mesh refinement is shown in Fig. 1. Based on the reference value method for the self adaptive technique, the minimum number of elements can be obtained under the requirement of error tolerance. Thus, the measure of error must be specified. Although many popular adopted measure of error have been used, e.g.,

1. Pointwise error,

2. Max norm: $\left\|u-u_{h}\right\|=\max _{a \leq x \leq b}\left|u(x)-u_{h}(x)\right|$,

3. $L_{2}$ norm: $\left\|u-u_{h}\right\|=\left\{\int_{a}^{b}\left|u-u_{h}\right|^{2} d x\right\}$, and

4. Energy norm:

$$
\left\|u-u_{e}\right\|=\left\{\int_{a}^{b} \sum_{i=0}^{m}\left|\frac{d^{i} u}{d x^{i}}-\frac{d^{i} u_{e}}{d x^{i}}\right| d x\right\}^{\frac{1}{2}},
$$

the $L_{2}$ norm along the boundary in energy sense has been adopted in this paper. 


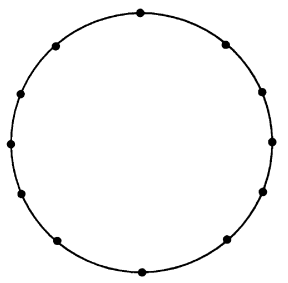

12 elements

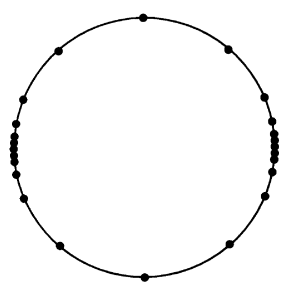

24 elements

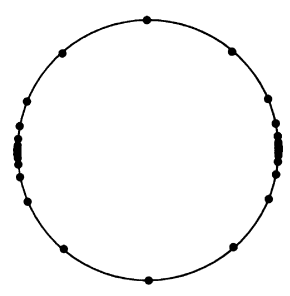

44 elements

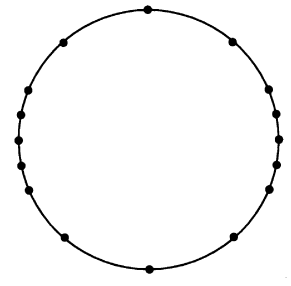

16 elements

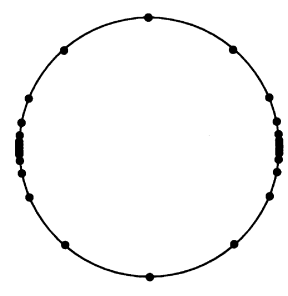

28 elements

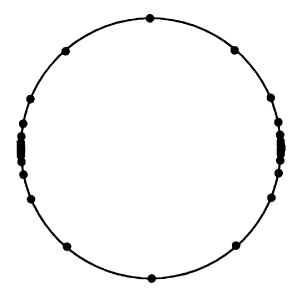

52 elements

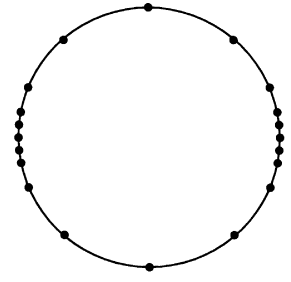

20 elements

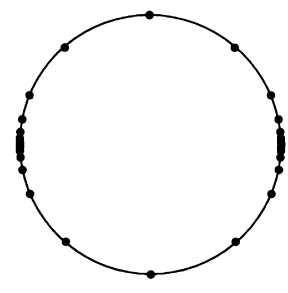

36 elements

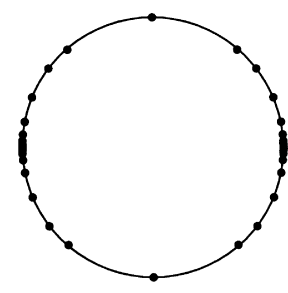

60 elements

Fig. 4. Nine types of adaptive mesh.

\section{Two numerical examples}

A benchmark example of test No. 2 in Boundary

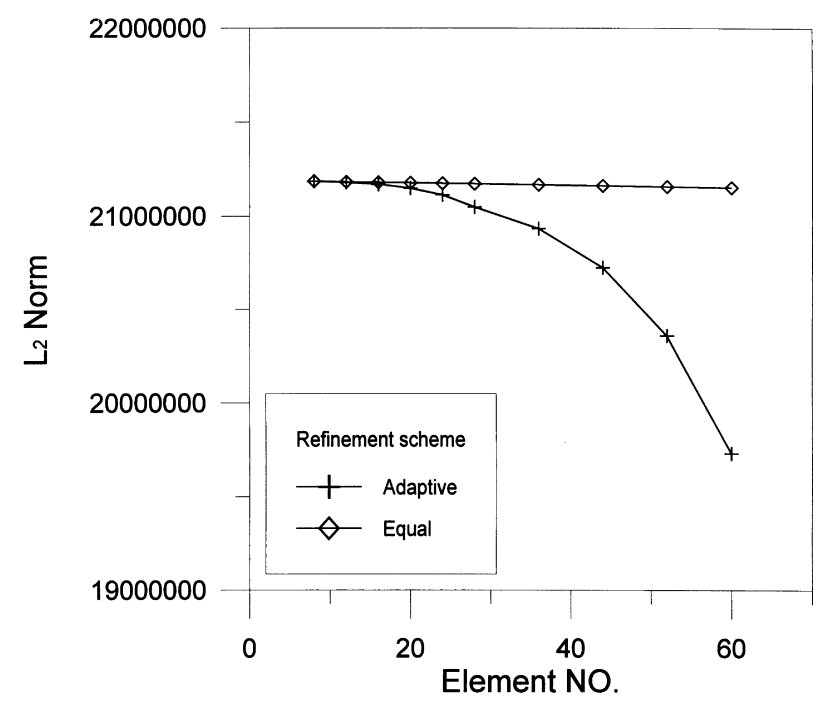

Fig. 5. The comparison of the error using the $L_{2}$ norm in different refinement schemes for adaptive mesh and equal mesh.
Elements Abstracts [21] is considered in Fig. 2. Chen et al. [22] have modified the interior program, BEPO2D, to solve the exterior problem. In this example, the geometrical shape is a unit circle governed by $x_{1}^{2}+x_{2}^{2}=1$.

The exterior problem satisfies the Laplace equation as follows:

$\nabla^{2} u(r, \theta)=0,1<r<\infty, 0<\theta<2 \pi$

with the boundary condition

$u(r, \theta)=f(\theta) \quad$ for $r=1$,

where

$f(\theta)=\left\{\begin{aligned} 1.0, & \text { if } 0<\theta<\pi \\ -1.0, & \text { if } \pi<\theta<2 \pi .\end{aligned}\right.$

The closed-form solution has been obtained as [13]

$u\left(x_{1}, x_{2}\right)=\frac{2}{\pi} \tan ^{-1}\left(\frac{2 x_{2}}{x_{1}^{2}+x_{2}^{2}-1}\right)$, 


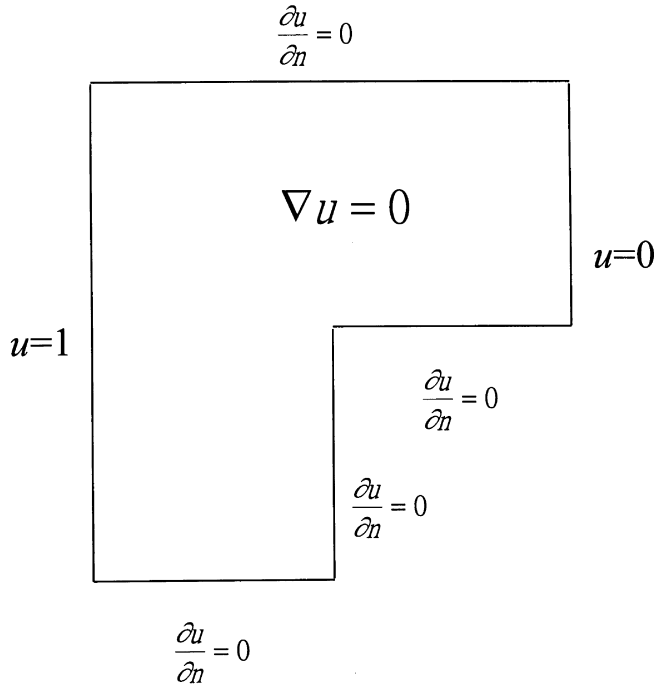

Fig. 6. L-shape problem.

and the potential gradients are

$$
\begin{aligned}
& \frac{\partial u}{\partial x_{1}}=\frac{2}{\pi} \frac{-4 x_{1} x_{2}}{\left(x_{1}^{2}+x_{2}^{2}-1\right)^{2}+4 x_{2}^{2}} \\
& \frac{\partial u}{\partial x_{2}}=\frac{2}{\pi} \frac{2\left(x_{1}^{2}+x_{2}^{2}-1\right)-4 x_{2}^{2}}{\pi\left(x_{1}^{2}+x_{2}^{2}-1\right)^{2}+4 x_{2}^{2}} .
\end{aligned}
$$

The initial mesh is divided into 16 elements. Bases on the Dual Boundary Element Method (DBEM) and using the BEPO2D program, which use the constant element scheme, the error estimators can be found. The six types of error estimators are shown in Fig. 3. The main feature of this Fig. 3 is that the six types of error estimators can predict a consistent shape of the error curve. This figure also shows that any one of the six kinds of error estimators can be adopted as an error indicator in the refinement scheme because the analytical solution obtained from the prescribed boundary value is utilized in $\left|t_{U T}(x)-t_{e}(x)\right|$, which is one of the six types of error estimators. For simplicity, only one error estimator is considered. Based on the adaptive technique, the nine element meshes are shown in Fig. 4. This result indicates that the adaptive technique can be successfully applied to find the optimum element mesh of the problem. In Fig. 3, $\left|t_{U T}(x)-t_{L M}(x)\right|$ is employed as an error indicator to construct the refinement scheme. Moreover, the measure of error using the $L_{2}$ norm by both the adaptive technique and the equal mesh tactics are shown in Fig. 5. These results indicate that a better convergence for adaptive refinement can be obtained compared to equal mesh refinement.

In order to extend the Dirichlet problem to mixed type problem, a L-shape interior problem is considered. The governing equation and boundary condition are shown in Fig. 6. The initial mesh is divided into 6 elements. Based on the dual formulation, the error tracking curve can be obtained from $\left|u_{U T}(x)-u_{L M}(x)\right|$ and $\left|t_{U T}(x)-t_{L M}(x)\right|$ in Fig. 7, which is one of the reliable error estimators. Using adaptive technique, mesh of adaptive iteration process are shown in Fig. 8. Further, the measure of the error using $L_{2}$ norm by both adaptive technique and equal mesh tactics is shown in Fig. 9. The result also indicates that a better convergence for adaptive technique can be obtained than uniform mesh does.

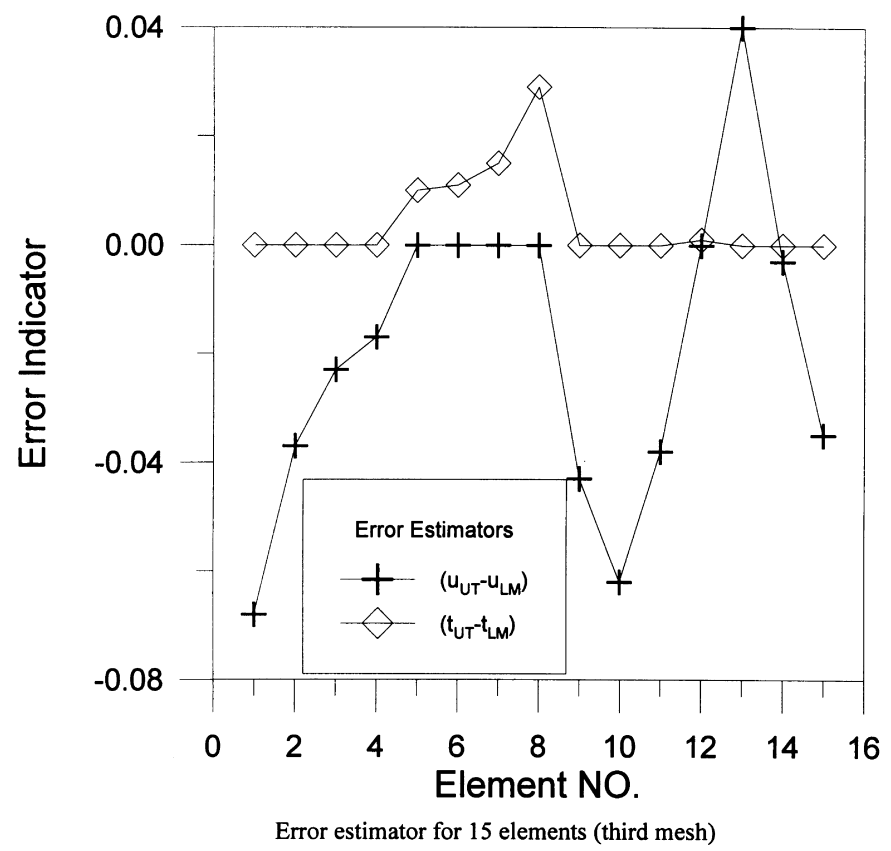

Fig. 7. Error tracking curve for L-shape problem. 

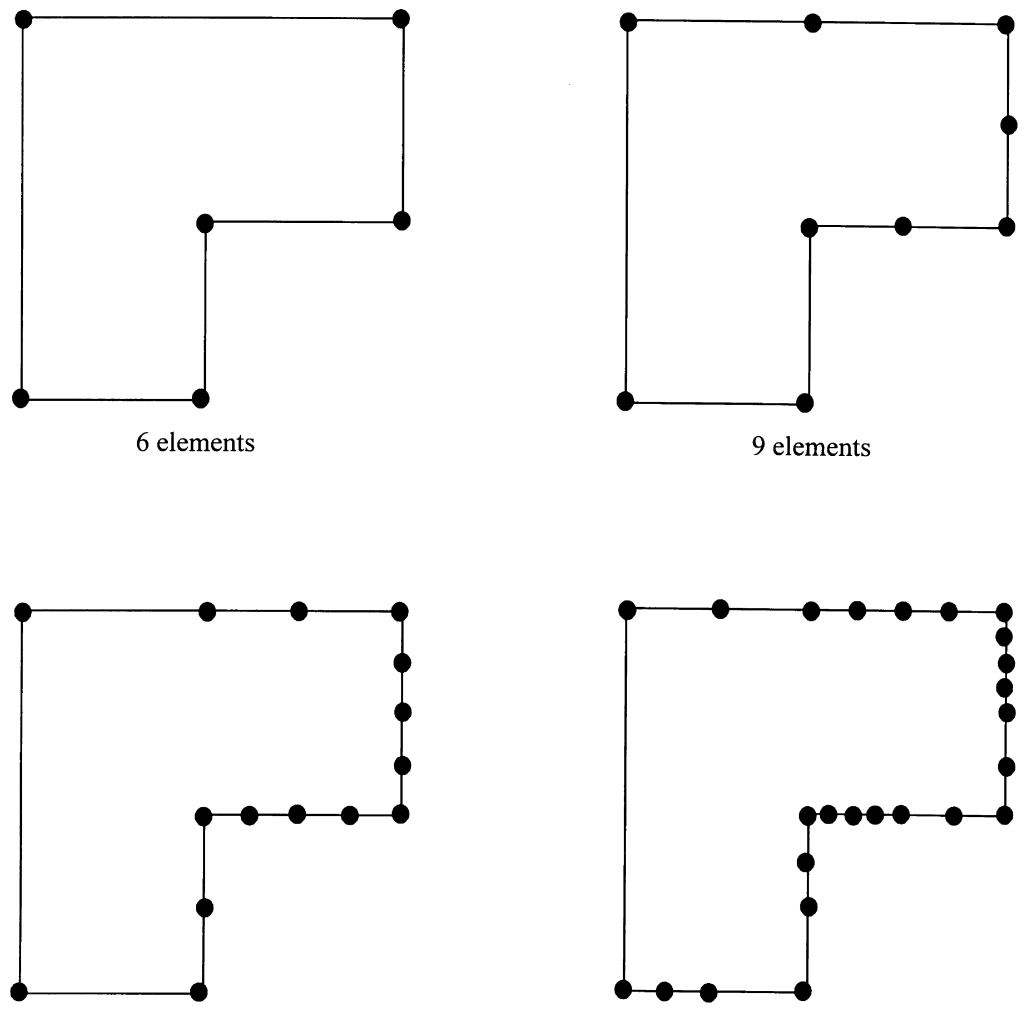

15 elements

23 elements

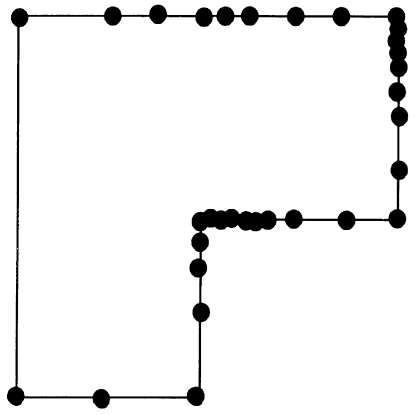

31 elements

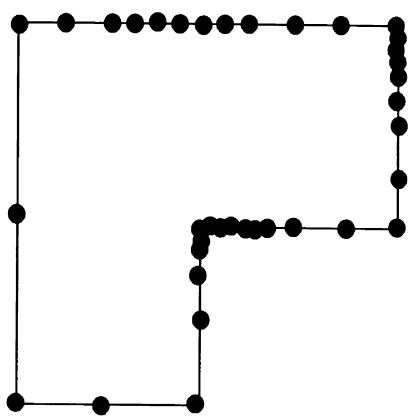

42 elements

Fig. 8. Adaptive iteration process for L-shape problem.

\section{Conclusions}

The dual boundary element method has been applied to construct the error estimators. Six types of error estimators have been described for tracking the pointwise error. $\left|u_{U T}(x)-u_{L M}(x)\right|$ and $\left|t_{U T}(x)-t_{L M}(x)\right|$, are suggested to be adopted in this paper, since they can be obtained directly from DBEM. Thus, error estimation has been successfully implemented by using DBEM. The exact error, $E_{\mathrm{u}}(x)$ and $\mathrm{E}_{\mathrm{t}}(x)$, defined by Paulino et al. [8,9] has been proven to be
$\left|u_{U T}(x)-u_{L M}(x)\right|$ and $\left|t_{U T}(x)-t_{L M}(x)\right|$, respectively. The value of $E_{\mathrm{u}}(x)$ or $\mathrm{E}_{\mathrm{t}}(x)$ is really not an exact error, since exact solution is not compared with; however, it is also another kind of error estimator in discrete system. The exact error is difficult to define. An exterior problem with two singular points has been used to show the faster convergence obtained using the adaptive mesh which is one of six error estimators, as compared to the convergence obtained using equal mesh discretization. Also, a mixed type L-shape problem was considered. 


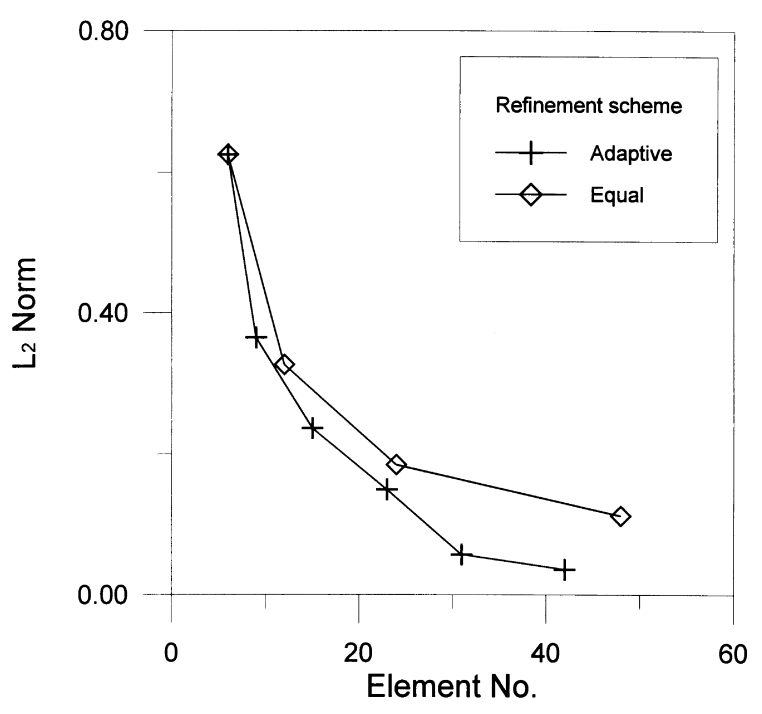

Fig. 9. The comparison of the error using the $L_{2}$ norm in different refinement schemes for adaptive mesh and equal mesh in L-shape problem.

\section{References}

[1] Rencis JJ, Jong KY. Error estimation for boundary element analysis. Journal of Engineering Mechanics. ASCE 1989;115:1993-2000.

[2] Gupta OP, Babu SS. Error analysis in applying a boundary element method for three-dimensional steady state potential problems. Computers and Structures 1996;58:289-297.

[3] Leal RP Mota Soares, CA. Adaptive boundary element method for bidimensional elasticity. Computers and Structures 1988;30:841-844.

[4] Kamiya, N, Aikawa, Y, Kawaguchi, K. Adaptive boundary element for the problem with mixed boundary condition. In: Brebbia CA, Kim, S, Osswald, TA, Power, H, editors. Boundary Elements XVII, 1995.

[5] Kita E, Kamiya N. Recent studies on adaptive boundary element methods. Advances in Engineering Software 1994;19:21-32.

[6] Carstensen C, Estep D, Stephen P. h-adaptive boundary element schemes. Computational Mechanics 1995;15:372-83.

[7] Zarikian, V, Gray, LJ, Paulino, GH. Pointwise error estimates for boundary element calculations. In: Brebbia, CA, Kassab, AJ, editors. Boundary element technology IV. Computational Mechanics Publications, 1994:253-60.

[8] Paulino, GH, Gray, LJ, Zarikian, V. A posteriori pointwise error estimates for the boundary element method, OAK Ridge National Laboratory, ORNL/TM-12820, 1995.

[9] Paulino GH, Gray LJ, Zarikian V. Hypersingular residuals- a new approach for error estimation in the boundary element method. International Journal for Numerical Methods in Engineering 1996;39:2005-2029.

[10] Hsiao, GC, Kleinman, RE, Li, R-E, Van den Berg, PM. Residual error - a simple and sufficient estimate of actual error in solutions of boundary integral equations. In: Gill, S, Brebbia, CA, Cheng, AHD, editors. Computational Engineering with Boundary Element, 1990;1:73-83.

[11] Kelly DW, Mills RJ, Reizes JA, Mills AD. A posteriori estimates of the solution caused by discretization in the finite element, finite difference and boundary element methods. International Journal for Numerical Methods in Engineering 1987;24:1921-1939.

[12] Rencis JJ, Hopkins DA, Chamis CC. Local and global accuracy estimates for boundary element method. Finite Elements in Analysis and Design 1991;9:229-245.

[13] Rencis JJ, Mullen RL. Solution of elasticity problems by a self adaptive mesh refinement technique for boundary element computation. International Journal for Numerical Methods in Engineering 1986;23:1509-1527.

[14] Ingber MS, Mitra AK. Grid optimization for the boundary element method. International Journal for Numerical Methods in Engineering 1986;23:2121-2136.

[15] Jayakumaran S, Hoole S. Perturbations in the adaptive refinement of boundary elements. Journal of Applied Physics 1988;63:3013-3015.

[16] Guiggiani M. Error indicator for adaptive mesh refinement in the boundary element method - a new approach. International Journal for Numerical Methods in Engineering 1990;29:1247-1269.

[17] Sun W, Zamani NG. Adaptive mesh redistribution for boundary element method in elasticity problems. Computers and Structures 1990;36:1081-1088.

[18] Cerrolaza MY, Alarcon E. p-adaptive boundary elements for three dimentional potential problems. Computer Applied Numerical Method 1987;3:335-345

[19] Chen, JT, Hong, HK. Boundary Element Method, 2nd Ed., New World Press, Taipei, Taiwan, (in Chinese), 1992.

[20] Chen JT, Hong HK, Chyuan SW. Boundary element analysis and design in seepage flow problems with sheetpiles. Finite Elements in Analysis and Design 1994;17:1-20.

[21] Test No. 2, Two dimensional steady state heat condition with a circular boundary in an infinite domain. Boundary Element Abstracts, 1990, 1, 90-91.

[22] Chen JT, Liang MT, Yang SS. Dual boundary integral equation for exterior problems. Engineering Analysis with Boundary Elements 1995;16:333-340. 